\title{
Penetrating keratoplasty for treatment of corneal abscesses in horses - case report
}

\author{
Zita Makra ${ }^{1}$, Imre Biksi ${ }^{1}$, Gábor Bodó \\ ${ }^{1}$ Szent István University, Veterinary Faculty, Department of Large Animal Clinical Sciences, Üllö, Hungary \\ ${ }^{2}$ Vetsuisse-Fakultät, Agroscope Swiss Institute of Equine Medicine, Bern, Switzerland
}

Received December 8, 2012

Accepted June 19, 2013

\begin{abstract}
This case report describes the surgical technique and outcome of allograft transplantation to treat full thickness corneal abscesses in three horses. In all three cases penetrating keratoplasty was performed. Each affected eye had an abscess within the corneal stroma. An initial full thickness circular corneal incision was made around the lesion. After excision of the stromal abscess the created defect was replaced with a larger diameter full thickness donor graft and the initial corneal incision was repaired. All of the horses that underwent penetrating keratoplasty procedure healed with a scar at the graft site and remained visual. Penetrating keratoplasty is an effective technique for surgical removal of medically nonresponsive full thickness stromal abscess in horses and results in a visual and cosmetically acceptable globe. The advantages of this technique compared to medical therapy are that there is less scarring, shorter healing times and less damage of the globe because of the concurrent uveitis. To the authors' knowledge, clinical experience with penetrating keratoplasty in horses has not been reported in Europe so far.
\end{abstract}

Equine, corneal transplant, stromal abscess, uveitis

Full thickness corneal abscess is a real challenge for the treating veterinarian and needs surgical repair. Penetrating keratoplasty (PK) has been described in the horse for full thickness stromal abscess (SA) (Andrew 2000; Plummer 2008; Brooks 2008, 2010). Corneal transplantation for treatment of severe inflammatory keratopathies has been performed successfully in horses at the University of Florida since 1993 (Brooks 2008). Corneal SA in the horse is a major disease that has the potential to render a previously normal eye extremely painful and blind (Plummer 2008). A SA may develop during the healing of a minor corneal defect if the surrounding epithelial cells migrate and divide over the defect, thus sealing microorganisms within the stroma (Hendrix 1995). The disease consists of two major components: the corneal lesion itself, which is an accumulation of inflammatory cells and debris with or without an infectious agent or foreign body, and the secondary uveitis that follows the corneal insult (Andrew 2010). Causative agents cannot be isolated in all cases, however, the majority of the cases are caused by fungus, gram-positive cocci, or gram-negative rods. Clinically it is a yellow-white stromal infiltrate of variable size and depth with corneal oedema, neovascularization and iridocyclitis (Hamilton 1994). If medical therapy alone is insufficient or the SA is situated deep within the stroma or results in severe iridocyclitis, a surgical approach is indicated. Surgical management has been associated with a more rapid recovery time and better visual outcome (Hendrix 1995). There is a lack of case reports from European clinics. Although fungal keratitis and SA are not rare in European horses, PK surgeries have not been described in complicated clinical cases so far in Europe.

The aim of this article was to describe the adequate technique and common complications of penetrating keratoplasty.

Address for correspondence:

Zita Makra

Department of Large Animal Clinical Sciences

Veterinary Faculty, Szent István University

Phone: +36-30-605-3472

Üllö, Dóra major, 2225, Hungary 


\section{Materials and Methods}

Three clinical cases of equine corneal abscesses were referred to the Large Animal Clinic of the Veterinary Faculty, Szent Istvan University, Hungary in the years 2010-2012. Horse profiles are described in Table 1. The first case was a dressage sport pony, the second and third cases were hobby horses. All three horses were presented for evaluation of profound ocular pain with corneal opacity that had been present prior to referral for 14-28 days, and treated with different antimicrobials and antiinflammatory drugs by the referring veterinarian, although without improvement.

Table 1. Data of the three horses with the corneal abscesses

\begin{tabular}{cccccccc}
\hline Case & Sex & $\begin{array}{c}\text { Age } \\
\text { (years })\end{array}$ & Breed & Eye & $\begin{array}{c}\text { Lesion location } \\
(\mathrm{mm})\end{array}$ & Size of lesion & Inciting incident \\
\hline 1 & Mare & 15 & Dutch pony & left & Temporal (4 o'clock) & 6 & Unknown \\
2 & Mare & 8 & Hungarian halfbred & left & Nasal (8 o'clock) & 8 & Trauma, ulcer \\
3 & Gelding & 11 & Hungarian halfbred & left & Ventral (6 o'clock) & 15 & Unknown \\
\hline
\end{tabular}

\section{Clinical findings}

In all three cases severe blepharospasm and epiphora were noted in the left eye. Direct pupil light reflex (PLR) and menace were present in both eyes except for case 3 in which only PLR was present in the left eye. The affected eyes were examined with slit lamp biomicroscopy and direct ophthalmoscopy, revealing a single 6-15 $\mathrm{mm}$ diameter focal yellow-white spot in the cornea that extended the entire thickness of the stroma. There were no fluorescein stain uptakes. The SA ruptured into the anterior chamber in case 3 resulting in hypopyon, miosis and neovascularisation with corneal oedema. Other structures of the globes and the contralateral eyes were normal. All 3 horses were diagnosed with full thickness corneal abscess with concurrent iridocyclitis in the left eye (Plate VIII, Fig. 1). In our cases, the location of the lesion, acute inflammatory signs and lack of compatible history made corneal neoplasia, parasitic lesions, granulation tissue, leukoma, corneal degeneration and scar tissue less likely as differential diagnoses. The lack of fluorescein uptake ruled out ulcerative keratitis. Stromal abscesses with or without a retained corneal foreign body were the most probable differentials.

\section{Medical and surgical treatment}

The decision to pursue surgical removal of the lesions was based upon their depth and the persistence of secondary uveitis despite medical therapy. All horses received the following medication prior to surgery: penicillin-streptomycin i.m. (Tardomyocel Comp. III. inj.) and flunixin meglumine $1 \mathrm{mg} / \mathrm{kg}$ i.v. once daily. Topical premedication included atropine ( $2 \%$ Atropine eyedrop FoNoVet) and chloramphenicol (Chloramphenicol $0.5 \%$ eyedrop FoNoVet) q6h. Under general anaesthesia the affected eye was cleaned and prepared routinely. After retrobulbar block and topical anaesthesia ( $0.4 \%$ oxybuprocain, Humacain eyedrop, TEVA Pharmaceuticals), the size of the abscess was measured with a caliper. The PK was performed as described by Brooks (2005a). In case 3 , because of the large extent of the lesion, a manual clear-cut was obtained with corneal scissors. Viscoelastic material (hyaluronate sodium, $10 \mathrm{mg} / \mathrm{ml}$, Hylartin V $2 \mathrm{ml}$, Pfizer Pharmaceutical Company) was injected into the anterior chamber through the perforation site to keep the eye inflated. Donor cornea was harvested from a fresh eyeglobe of a horse in case 1 and kept moistured in a refrigerator for one day, until utilization. In case 2 and 3 , frozen eyeglobes were used following thawing as donor corneas. The viscoelastic was not removed from the anterior chamber, and was reinflated with balanced salt solution (BSS) via limbal injection at the end of suturing. The corneal wound was checked for aqueous leakage with Seidel test. The eye had a conjunctival advancement pedicle flap placed over the PK incision in case 2 . A subconjunctival injection of $1 \mathrm{mg}$ atropine sulphate $1 \%$, and placement of a subpalpebral lavage system (SPL, Equivet ocular lavage 8 Fr kit, Kruuse Veterinary Products) were the last steps of the surgery. A temporary tarsorrhaphy was used to protect the large surgical site in case 3 for 6 days. The diseased keratectomy buttons from the operated eyes were submitted for aerobic bacterial and fungal culture, sensitivity; cytology and histopathology.

\section{Postoperative treatment and management}

Postoperative medication was continued as before surgery for 6 days. Systemic antibiotic drug was used prophylactically due to the necessity of entering the globe during surgery or rather the possible infectious cause. Topical therapy was continued through SPL with the addition of autologous serum (every four hours a day) to decrease tear film proteolytic activity, and an eye protective hood was put on for the first week. We also added topical $2 \%$ povidone iodine solution twice daily via SPL, and natamycine every six hours a day (Pima-Biciron $\mathrm{N}$ ophthalmic ointment, Infectopharm Pharmaceuticals) in all 3 cases because we suspected fungal origin of the keratitis based on the clinical examination and progress. Topical chloramphenicol was continued every four hours a day for 4 weeks postoperative, and then either tapered or discontinued. In case 2, topical ciprofloxacin (Ciloxan 
$3 \mathrm{mg} / \mathrm{ml}$ eyedrop, Alcon Products) was applied from the beginning and it showed to be indicated based on culture and sensitivity testing results. As healing progressed medications were tapered on an individual basis as required. Over the next 7 days, as the pupil became more dilated and the hypopyon consolidated, corneal vessels to the graft and some resolving corneal oedema were found. The horses were hospitalized for 25 days (case 1), 12 days (case 2), and 6 days (case 3) and were rechecked on daily basis while hospitalized. The horses were discharged with indwelling SPLs and were on topical medications (chloramphenicol/ciprofloxacin, atropine, serum, in Case $310 \%$ diluted Aloe Vera solution, Aloe First, Forever Living Products). After discharge from our facility they were re-examined every 7 days by the referring veterinarians. Topical therapy via SPL was continued for 38 days in case 1, 28 days in case 2 and 42 days in case 3, with an average of 36 days. The subpalpebral ocular lavage catheter (SPL) was removed when there was no further evidence of active disease.

\section{Results}

The surgery time for PK was 100, 70 and 90 min in case 1, 2 and 3, respectively, with an average of $86 \mathrm{~min}$, which was similar to the cases $(91 \mathrm{~min})$ described in a previous study (Whittaker 1997). No complications were observed during surgery and the treatment period. Corneal specimens from the excised abscesses were submitted for aerobic bacterial and fungal culture, sensitivity testing, cytology and histopathology. The keratectomy specimens demonstrated profound keratitis, the cellular components being mixed and varied from suppurative to histiocytic.

In Case 1 histopathology showed that the structure of the entire corneal epithelium, stroma and Descemet's membrane were severely disintegrated and diffusely infiltrated with inflammatory elements such as degenerated neutrophils, macrophages and lymphoid cells. In some deeper parts of the specimen angiofibroblast tissue proliferation with a lot of cellular elements was observed. Chronic inflammatory cell accumulation was present but neither foreign material nor pathogen agents with haematoxylin-eosin or PAS stains were detected.

In Case 2, cytology revealed that most of the numerous neutrophils had degenerative nucleus and phagocytosed coccoid bacterial agents. Several mononuclear phagocytes with variously differentiated epithelial cells were isolated. No fungal hyphae were detected by PAS staining.

In Case 3, all microscopic signs of severe necrotic inflammation of the entire cornea were found with masses of fungal hyphae detected by Romanowsky staining (Fig. 2). Aerobic cultures revealed Staphylococcus aureus (Case 1) sensitive to chloramphenicol and betahaemolytic Streptococcus equi (Case 2) sensitive to ciprofloxacin. Fungal cultures yielded no growth in any of the samples.

Long-term follow-up (3-18 months) was carried out through telephone conversations between the owner and the referral veterinarians. Dense fibrosis was evident at the graft site and in the area of conjunctival graft placement (Case 2), however, a return to full athletic function was achieved in all three horses. Case 1 and 2 had positive menace response and there was no aqueous flare, the pupils were symmetrical. Case 1 was operated more than 2 years ago, and the horse was sold to Germany 1 year after PK, so the animal was lost to long-term follow-up. In Case 3 the purpose of the surgery was to save the eye and avoid enucleation. Three months post operation this horse had a comfortable eye and some light perception but without menace response.

\section{Discussion}

Following a corneal microtrauma the epithelial wound can heal quickly while sealing microbes or foreign body in the deep. This can result in drawing neutrophils to the site of infection and in visible corneal infiltration, opacity, or abscess (Plummer 2008). A corneal ulcer was mentioned in the history only in Case 2. Especially fungal elements have an affinity for the Type VI collagen of Descemet's membrane, so DSA should be 
considered to be of a fungal aetiology (Gaarder 1998). In our cases all three horses had been treated before referral without success, furthermore the location and extent of the lesions warranted the surgery. Penetrating keratoplasty in the management of stromal abscessation was previously described and positive visual results were obtained (Hendrix 1995; Whittaker 1997). Either fresh or frozen donor cornea can be utilized in PKs. Frozen corneas could be stored for 6 months (Brooks 2008). In Case 1, we were fortunate enough to use a fresh graft, but frozen ones are easier to access at any time. The most practical way to get donor corneas is storing the whole globe in a deep-freezer; frozen corneas are easier to handle if not completely thawed at utilization. The epithelium of the donor graft often sloughs leaving it open to infection and excessive tear film proteinase activity. This is why we used autologous serum to block proteinases in our cases; placing a conjunctival flap as we did in Case 2, where the surrounding cornea was much degraded. Penetrating keratoplasty patients were probably guaranteed graft rejection but it was deemed necessary to support the weakened tissue surrounding the lesion. On this occasion the function of the corneal graft was tectonic. We could successfully utilize both fresh and frozen corneas in our cases and they were equally incorporated in the operated eyes. Graft rejection and scar formation have been unavoidable with PK. As with any corneal transplant, 3-5 days after implantation, the graft begins to swell as part of the rejection initiated by the host. The donor graft becomes vascularized as the host incorporates the donor tissue at 5-7 days post operation (Trbolova 2003; Brooks 2008). Our cases were more severe in nature with significant preoperative corneal destruction, worse uveitis, and absence of normal superficial corneal stroma, therefore requiring PK surgery. In the most severe one in Case 3 , the abscess perforated into the anterior chamber causing hypopyon and miosis.

After clinical examination and cytology results, topical antifungal medications were initiated postoperatively. Antifungal solution that can be effective and instilled via SPL is not available in our country so the only treatment was the use of natamycine eye ointment. Routine usage of systemic antifungals should be considered postoperatively in these cases but the high cost stands against it. Examination of the specimens confirmed that Case 1 and 2 had bacterial, and Case 3 fungal cause, so intensive antibiotic and antifungal treatment was indicated. Animals were considered healed when they required no further treatment. In our cases the healing time was 36 days, shorter than in another study, where it was 57 days (Whittaker 1997). Cases 1 and 2 had successful outcomes with comfortable visual eyes. Successful healing and maintenance of vision were reported in $75 \%$ and $100 \%$ of horses undergoing PK (Whittaker 1997; Brooks 2005a). The visual outcome was $77.9 \%$ and the average time for complete healing 46.7 \pm 23.1 days in another study (Brooks 2008). Corneal ulceration or malatia, incisional dehiscence or aqueous leakage requiring surgical repair, secondary infections at the surgery site, synechiae formation, focal nonprogressive cataract formation, lens capsule rupture intraoparately associated with PK are reported complications that also contribute to a prolonged treatment. Blindness from corneal scarring, phthisis, or pupil occlusion $(n=13)$, and enucleation $(n=5)$ occurred in 18/86 cases $(20.9 \%)$ that had PK (Denis 2004; Brooks 2005b, 2008).

Limitations of the study are that we have had only three cases within the last two years. In summary, we can say that in the three cases reported in this study, medically nonresponsive eyes that would have been lost otherwise were saved with allograft transplantation. We used thicker suture material (6/0 Vicryl), no systemic antifungal, and tried topical 10\% aloe vera solution postoperatively that had not been mentioned previously (Brooks 2008). Stromal abscess is a major disease process in the horse not only in America, but also in Europe. In most cases it requires surgical intervention to achieve the best result. Corneal transplantation is a very useful surgical intervention in the horse that needs more publicity, because it is associated with high success rates, good visual outcomes and shorter treatment times. 


\section{References}

Andrew SE, Brooks DE, Biros DJ, Denis HM, Cutler TJ, Gelatt KN 2000: Posterior lamellar keratoplasty for treatment of deep stromal abscesses in nine horses. Vet Ophthalmol 3: 99-103

Andrew SE, Willis AM 2010: Diseases of the cornea and sclera. In: Gilger BC (Ed.): Equine Ophthalmol. Elsevier Saunders Publishing, St. Louis, MO, pp. 157-251

Brooks DE 2005a: Penetrating keratoplasty, deep lamellar endothelial keratoplasty, and posterior lamellar keratoplasty in the horse. Clin Tech Equine P 4: 37-49

Brooks DE 2005b: Equine stromal and endothelial keratopathies: Medical management of stromal abscesses, eosinophilic keratitis, calcific band keratopathy, striate band opacities, and endotheliitis in the horse. Clin Tech Equine P 4: 21-28

Brooks DE 2008: Complications of ophthalmic surgery in the horse. Vet Clin N. Am-Equine 24: 697-734

Brooks DE 2010: Targeted lamellar keratoplasty in the horse: A paradigm shift in equine corneal transplantation. Equine Vet J 37: 24-30

Brooks DE, Matthews AG 2007: Equine ophthalmology. In: Gelatt KN (Ed.): Veterinary Ophthalmol. Blackwell Publishing, Ames, IA, pp. 1165-1274

Brooks DE, Plummer CE, Kallberg ME, Barrie KP, Ollivier FJ, Hendrix DV, Baker A, Scotty NC, Utter ME, Blackwood SE, Nunnery CM, Ben-Shlomo G, Gelatt KN 2008: Corneal transplantation for inflammatory keratopathies in the horse: Visual outcome in 206 cases (1993-2007). Vet Ophthalmol 11: 123-133

Denis HM 2004: Equine corneal surgery and transplantation. Vet Clin N Am-Equine 20: 361-380

Gaarder JE, Rebhun WC, Ball MA, Patten V, Shin S, Erb H 1998: Clinical appearances, healing patterns, risk factors, and outcomes of horses with fungal keratitis: 53 cases (1978-1996). J Am Vet Med Assoc 213: $105-112$

Hamilton HL, McLaughlin SA, Whitley EM, Gilger BC, Whitley RD 1994: Histological findings in corneal stromal abscesses of 11 horses: Correlation with cultures and cytology. Equine Vet J 26: 448-453

Hendrix DVH, Brooks DE, Smith PJ, Gelatt KN, Miller TR, Whittaker C, Pellicane C, Chmielewski N 1995: Corneal stromal abscesses in the horse: a review of 24 cases. Equine Vet J 27: 440-447

Plummer CE, Kallberg ME, Ollivier FJ, Barrie KP, Brooks DE 2008: Deep lamellar endothelial keratoplasty in 10 horses. Vet Ophthalmol 11: 35-43

Trbolová A, Kozák I, Kolodzieyski L, Juhás T, Ledecký V 2003: Keratoplasty á Chaud, Limbal Autotransplantation and Transplantation of Amniotic Membrane in Management of Severe Chemical Burns of the Eye. Acta Vet Brno 72: 571

Whittaker CJG, Smith PJ, Brooks DE, Hendrix DV, Chmielewski NT, Andrew SE, Gelatt KN 1997: Therapeutic penetrating keratoplasty for deep corneal stromal abscesses in eight horses. Vet Comp Ophthalmol 7: 19-28 


\section{Plate VIII}

Makra Z. et al.: Penetrating ... pp. 303-307

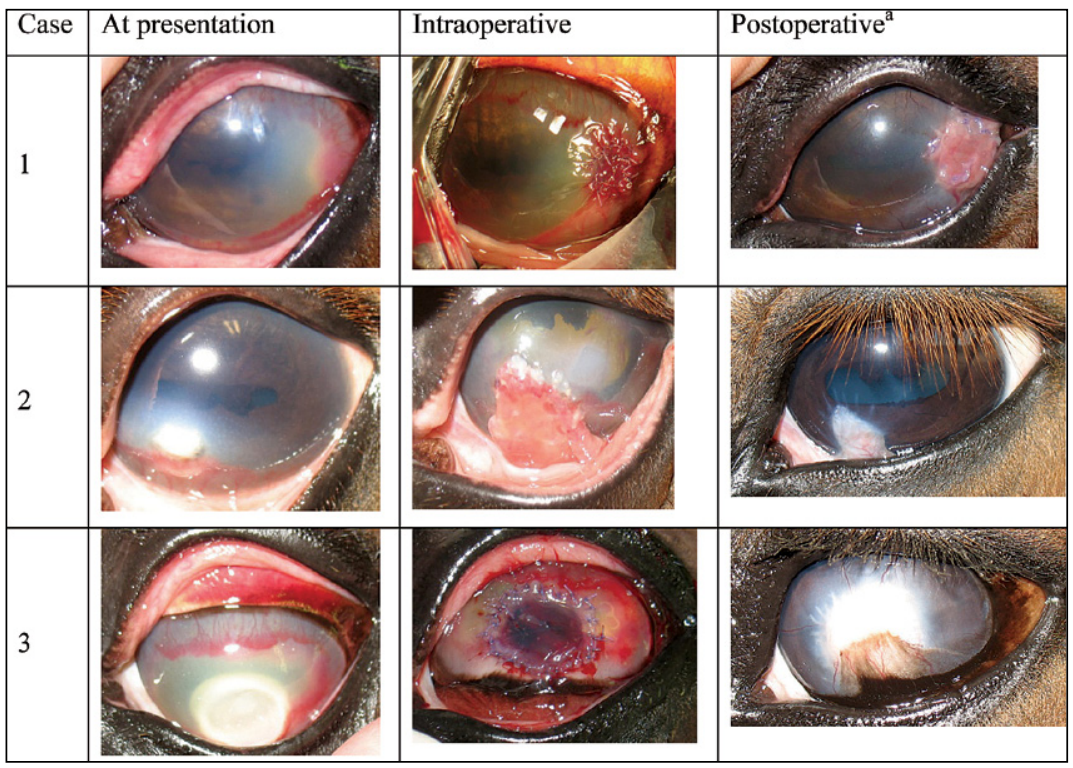

${ }^{\text {aCCase }} 1$ - 1 month postoperative, case 2 - 18 months postoperative, case 3 - 3 months postoperative

Fig. 1. Clinical progress of the cases documented with photos 